\title{
Patient's quality of life after surgery and radiotherapy for extremity soft tissue sarcoma - a retrospective single-center study over ten years
}

Rebekka Götzl ${ }^{1,2^{*}}$ (D), Sebastian Sterzinger ${ }^{1}$, Sabine Semrau ${ }^{3}$, Nikolaos Vassos ${ }^{4}$, Werner Hohenberger ${ }^{4}$, Robert Grützmann ${ }^{4}$, Abbas Agaimy ${ }^{5}$, Andreas Arkudas ${ }^{1}$, Raymund E. Horch ${ }^{1}$ and Justus P. Beier ${ }^{1,2}$

\begin{abstract}
Background and objectives: The purpose of this study is to analyze major complication rates and different aspects of health-related quality of life (HRQoL) in extremity soft tissue sarcoma (STS) patients treated with or without radio (chemo) therapy and surgery.

Methods: We performed a retrospective analysis of all patients who underwent Extremity STS excision from 2004 to 2014 (182 patients included). Patients' data were collected from patients' records. HRQoL was assessed by using EORTC QLQ-C30.

Results: A total of 182 patients underwent sarcoma resection. After neoadjuvant radiochemotherapy (RCT), the major-complication rate amounted to $28 \%$ (vs. 7\%, no radiotherapy, $p<0.001$ ). Major-complication rates after adjuvant radiotherapy (RT) occurred in 8\% (vs. 7\%, no radiotherapy, $p=0.265$ ). Comparison QoL scores between treating with neoadjuvant RCT or without RT revealed significant worse scores with neoadjuvant RCT. Further stratification of disease control of these patients showed significant reduced scores in the group of disease-free patients with neoadjuvant RCT compared to irradiated disease-free patients.
\end{abstract}

Discussion: To date, there have only been a few investigations of QoL in STS. Retrospective study on quality of life have limitations, like a lack of baseline evaluation of QoL. Patient candidated to radiation therapy could have had worse QoL baseline due to more advanced disease. Disease status of the patients who answered the questionnaires could have been an influence of QoL and we could show reduced scores in the group of disease-free patients with neoadjuvant RCT, but not for the patients with recurrence or metastasis, so it is very hard to discriminate whether radiation therapy could really have an impact or not.

Conclusion: This study might assist in further improving the understanding of QoL in STS patients and may animate for prospective studies examining the oncological therapies impact on HRQoL.

Keywords: Soft tissue sarcoma, QLQ-C30, Sarcoma radiotherapy, Sarcoma major complication rates, Health related quality of life

\footnotetext{
* Correspondence: rgoetzl@ukaachen.de

${ }^{1}$ Department of Plastic and Hand Surgery, Comprehensive Cancer Center,

Universitiy Hospital of Erlangen, Friedrich-Alexander-University

Erlangen-Nürnberg (FAU), Erlangen, Germany

${ }^{2}$ Present Address: Department of Plastic Surgery, Hand and Burn Surgery,

University Hospital of Aachen, RWTH University of Aachen, Aachen, Germany

Full list of author information is available at the end of the article
}

(c) The Author(s). 2019 Open Access This article is distributed under the terms of the Creative Commons Attribution 4.0 International License (http://creativecommons.org/licenses/by/4.0/), which permits unrestricted use, distribution, and reproduction in any medium, provided you give appropriate credit to the original author(s) and the source, provide a link to the Creative Commons license, and indicate if changes were made. The Creative Commons Public Domain Dedication waiver (http://creativecommons.org/publicdomain/zero/1.0/) applies to the data made available in this article, unless otherwise stated. 


\section{Background}

Soft tissue sarcomas are rare and account for only $<1 \%$ of all malignancies. Modern sarcoma treatment is an interdisciplinary challenge. Besides surgery, playing the central role, radiotherapy and chemotherapy are other important treatment modalities. Historically, radical surgery including limb amputation was often used to achieve wide longitudinal margins. Studies performed in the 1970s and 1980s showed no influence on overall survival, when limb-conserving surgery and adjuvant radiotherapy are combined, compared with radical amputation alone [1].

Prospective and retrospective studies have suggested that radiotherapy improves the local control rates in the setting of resectable disease and the overall survival $[2,3]$. But, there is still a lack of survival impact of RT in STS, especially in extremity STS. Newer retrospective analyses showing an impact by RT on survival in STS are largely open to criticism [4]. However, many authors describe that wound complication rates are higher after preoperative irradiation, and long-term function is worse after postoperative irradiation, probably as a result of higher postoperative radiation doses, larger radiation fields, and resulting fibrosis $[5,6]$.

Multimodal treatment regimens might inflict a substantial morbidity and mortality with a substantial effect on health-related quality of life (HRQoL). When counseling patients on various treatment options, information of quality of life (QoL) following various types of treatment is therefore very important [7].

For a long time, soft tissue sarcoma patients had poor 5 -year survival rates below 50\%. With improvements in diagnosis and treatment, 5-year survival rate increased to $60-70 \%$ [5]. More patients with soft tissue sarcoma became long-term survivors and analysis of HRQoL should be much more important. However, little attention has been paid to this issue in the literature [8]. In particular, little is known about the role of RT for QoL in soft tissue sarcoma treatment.

The purpose of this study is to analyze different aspects of HRQoL in soft tissue sarcoma patients treated with or without radiotherapy and surgery over a period of 10 years at a single sarcoma center.

\section{Patients and methods}

\section{Patients}

We performed a retrospective review of all patients who underwent sarcoma excision from 2004 to 2014 at our University Hospital. Inclusion criteria for this analysis were histologically confirmed diagnosis of soft tissue sarcoma of the extremities. A total of 182 patients with different entities of soft tissue sarcoma were treated with surgery. Of these, 49\% were treated with neoadjuvant RCT and 7\% with adjuvant RT, respectively. Indications for preoperative RCT were large tumors, close margins and to avoid R1-resection; for postoperative radiotherapy, higher stage diseases, dedifferentiated tumors and close margins. Neoadjuvant radiotherapy was only applied as RCT. Postoperative RT was applied without chemotherapy. Patients with neoadjuvant and adjuvant radiotherapy (1\%, data not shown) as well as Patients treated with isolated hyperthermic limb perfusion were excluded, because of the very low number of patients treated that way rendering statistical analyses impossible. Data concerning patient characteristics, clinical variables, disease staging, and treatment outcomes were collected from the patient files and double-checked. There is a lack of data regarding precise tumor size in the majority of patients. However, size of excision is part of the data set recorded, so it was evaluated for this study as follows: we differentiated the median excision size into two groups: smaller than $10 \mathrm{~cm}$ or $10 \mathrm{~cm}$ and larger. For median size of excision, we analyzed the excision size in three diameters (length, width and height). From these data, we calculated the mean diameter and called it median size of excision.. Major complications are defined as complications which made an operative or in-hospital treatment necessary (Clavien-Dindo $\geq 3$ ). Operations and in-hospital treatments were counted $(1,2,3,>3)$. Tumor staging was performed according to the TNM classification and the FNCLCC Grading (G1-3) [9]. QoL questionnaires were sent to all included patients and, if answers to any of the given questions were missing, questionnaires were completed by telephonic interview.

\section{QLQ measures}

The HRQoL was assessed by using the core questionnaire of the European Organization for Research and Treatment of Cancer (EORTC QLQ-C30). The QLQ-C30 contains a global QoL scale, five function scales (physical, role, cognitive, emotional, and social), three symptom scales (fatigue, pain, and nausea/vomiting), and six single items (dyspnea, insomnia, appetite loss, constipation, diarrhea, and financial difficulties). All scores were linearly transformed such that they ranged from 0 to 100, in accordance with the EORTC Scoring Manual. A higher global QoL/health score equates to better overall QoL, and a higher score for functional scales corresponds to a better functioning, whereas higher score for a symptom scale indicates more symptoms [10]. HRQoL data about patients with adjuvant radiotherapy were excluded because of the small group size (3 patients).

\section{Statistics}

Calculations were performed using the Statistical Package for the Social Sciences (version 19.0, SPSS Inc., Chicago, IL, USA).

Survival, including possible influencing factors, was calculated using the Kaplan-Meier method with logrank tests (Mantel-Cox) (Fig. 1). Comparison of patient 


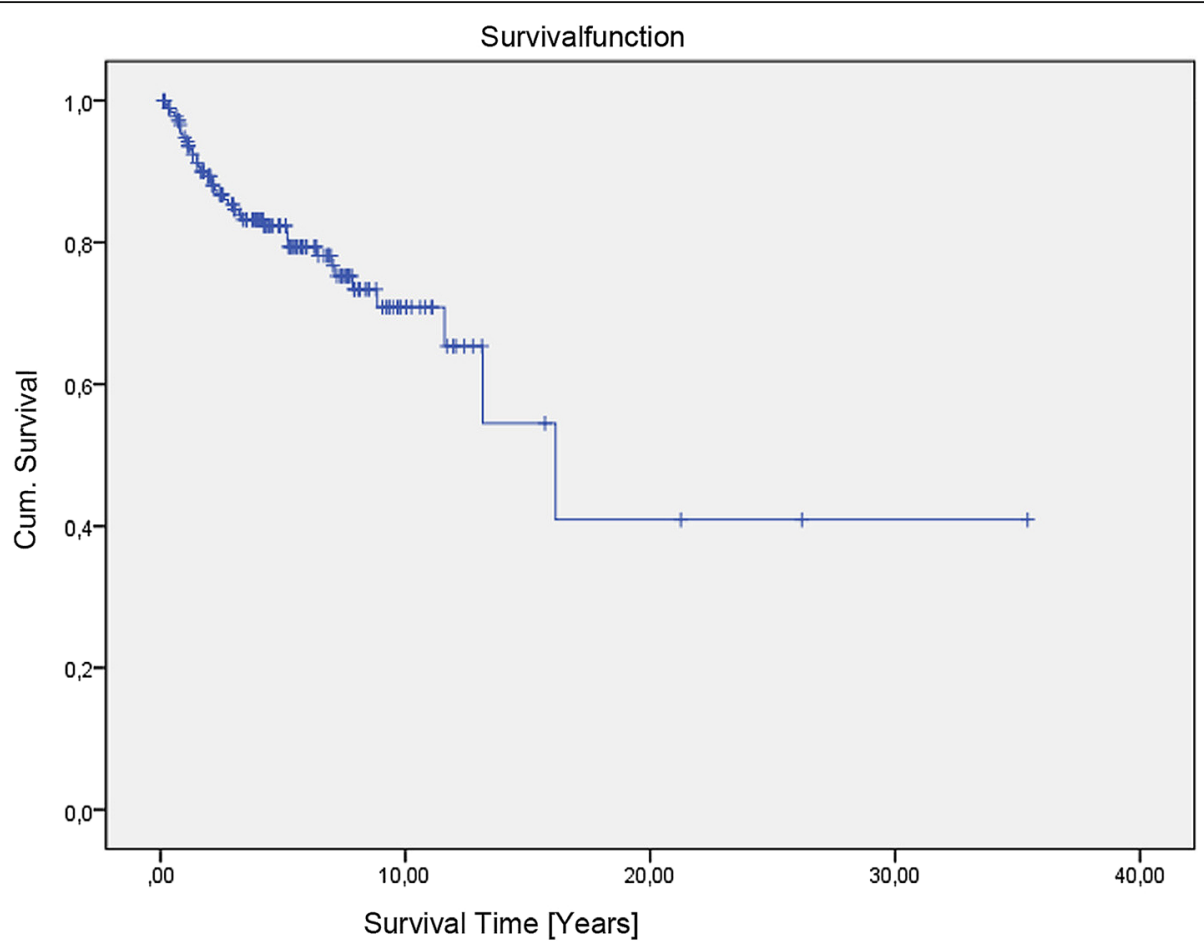

$\neg$ Survivalfunction

Fig. 1 Kaplan-Meier Curve of Survival Function of the 182 patients with Extremity STS

characteristics between the groups was performed by cross-tables and exact chi-square test, exact MannWhitney test, exact Fisher-test, and t-test for categorical, ordinal, and continuous variables, respectively. QoL data are presented as mean values and $95 \%$ confident interval. Comparisons between QoL scores for the groups were made by Shapiro-Wilk test and absent standard distribution by Wilcoxon test.

Additionally, LQ-Patients were stratified into four groups: Neoadj. RT or No RT and Disease-free, Neoadj. RT or No $\mathrm{RT}$ and Recurrence/Metastasis (further differentiation into a separate group recurrence and another group metastasis was because of the very low number of patients with recurrence or metastasis statistical impossible) and statistical significance were calculated using Kruskal-Wallis H-test and the subsequent pairwise comparison was performed using the Mann-Whitney U-test after Bonferroni procedure.

\section{Results}

From 2004 to 2014, a total of 182 patients underwent sarcoma resection of the extremities in the Department of Surgery or the Department of Plastic and Hand Surgery of our University Hospital. Liposarcoma was the primary diagnosis, followed by undifferentiated pleomorphic sarcoma (UPS) including synovial sarcoma and fibroblastic/myofibroblastic sarcoma. The distribution of the different sarcoma subtypes treated at our institution is illustrated in Table 1.
The overall survival rate after 5 years was $82 \%$ and after 10 years $71 \%$ (Fig. 1). The average age of the patients at the time of primary diagnosis of sarcoma was 58 years (range, 15-89), and the median follow-up time was 3.7 years. The majority of primary sarcoma was high-grade tumors (G2: 35\%, G3: 43\%). At definite operation on the primary sarcoma resection, in $89 \%$ complete resection was achieved with free surgical margins. In $8 \%$, the tumor was macroscopically removed, but histopathological evaluation revealed an R1-status. In four cases (2\%) only tumor mass debulking (R2) was performed. 49\% of all included soft tissue sarcoma patients were additionally treated with neoadjuvant RCT and adjuvant RT was used in $7 \%$. A summary of the data is given in Table 2 .

Postoperative major complication rates (Clavien-Dindo 23) are presented in Table 3. After neoadjuvant RCT, a major complication rate of $28 \%$ vs. $7 \%$ without RT $(p<0.001)$ was observed. Major complications after adjuvant RT occurred in $8 \%$ with no significant difference to patients without RT (7\%, not statistically significant, $p=0.265$ ). 93\% of patients with adjuvant RT had no major complications vs. $72 \%$ who had received neoadjuvant radiotherapy. However, in case of any major complications after adjuvant RT we noticed a minimum of two complications (vs. mostly one complication of patients with neoadjuvant radiotherapy). Major complications after chemotherapy (CT) only were not detected. 
Table 1 The proportions of the different sarcoma subtypes of all patients who underwent sarcoma excision from 2004 to 2014 in our University Hospital and of patients who answered lifequality quetionnaire. Subtypes were classified according to WHO classification

\begin{tabular}{lll}
\hline & $\begin{array}{l}\text { All Patients } \\
n=182\end{array}$ & $\begin{array}{l}\text { LQ-Patients } \\
n=70\end{array}$ \\
\hline Liposarcoma & $36(20 \%)$ & $16(21 \%)$ \\
Well differentiated & $21(11,5 \%)$ & $11(16 \%)$ \\
De- differentiated & $2(1 \%)$ & $2(3 \%)$ \\
Myxoid & $10(5 \%)$ & $2(3 \%)$ \\
$\quad$ Pleomorph & $3(2 \%)$ & $1(1 \%)$ \\
Undifferentiated pleomorphic sarcoma & $26(14 \%)$ & $7(10 \%)$ \\
Synovial sarcoma & $11(6 \%)$ & $5(7 \%)$ \\
Fibroblastic/myofibroblastic sarcoma & $43(24 \%)$ & $23(30 \%)$ \\
Undifferentiated sarcoma & $33(18 \%)$ & $9(12 \%)$ \\
Leiomyosarcoma & $13(7 \%)$ & $7(9 \%)$ \\
Rhabdomyosarcoma & $7(4 \%)$ & $2(3 \%)$ \\
Extraskeletal chondro-/ osteosarcoma & $5(3 \%)$ & $2(3 \%)$ \\
Malignant peripheral nerve sheath tumor & $4(2 \%)$ & $3(4 \%)$ \\
Angiosarcoma & $2(1 \%)$ & $0(0 \%)$ \\
Other unclassified sarcoma & $2(1 \%)$ & $1(1 \%)$ \\
\hline
\end{tabular}

Seventy patients answered the QoL-questionnaires and were included in the analyses. $23 \%$ of the patients died, $27 \%$ gave no feedback and $12 \%$ of the patients refused to attend. Median time between treatment and questionnaires was 65 months (95\% Confidence 59-72 months). All included patients reported that they fully understood the questionnaires. $56 \%$ of the patients were treated with neoadjuvant RCT and $44 \%$ of the patients were not irradiated. Comparison of QoL scores in the QLQ-C30 between the two groups with or without neoadjuvant RCT revealed significant differences in global QoL, in physical functioning, in role functioning, emotional functioning, social functioning, in fatigue, general pain and in financial problems (Table 4 and Fig. 2).

Stratification of Disease-control of the LQ-Patients into four groups (Neoadj. RT or No RT and Diseasefree, Neoadj. RT or No RT Recurrence/Metastasis) showed significant differences in physical functioning (pairwise comparison: Neoadj. RT + Disease-free vs. No $\mathrm{RT}+$ Disease free, $\left.\mathrm{H}=17.979, p=0.003^{*}\right)$, role functioning (pairwise comparison: Neoadj. RT + Disease-free vs. No RT + Disease free, $\left.\mathrm{H}=13.440, p=0,05^{*}\right)$, social functioning (pairwise comparison: Neoadj. RT + Disease-free vs. No $\mathrm{RT}+$ Disease free, $\mathrm{H}=17.904, p=0.002^{*}$ ) and in dyspnea (pairwise comparison: Neoadj. RT + Disease-free vs. Neoadj. RT + Recurrence/Metastasis, $\mathrm{H}=-25.588$, $p=0.02 \%$ Neoadj. RT + Recurrence/Metastasis vs. No $\mathrm{RT}+$ Recurrence/Metastasis, $\mathrm{H}=-28.250, p=0.05^{*}$; and, Neoadj. RT + Recurrence/Metastasis vs. No RT + Disease-free, $\mathrm{H}=-32.250, p=0.002 \%)$ and a strong trend in

Table 2 Patient and disease characteristics of 182 patients with soft tissue sarcoma which underwent sarcoma resection our University Hospital (All Patients) and of patients who answered life-quality questionnaire (LQ-Patients)

\begin{tabular}{|c|c|c|c|c|c|c|c|}
\hline & \multicolumn{4}{|l|}{ All Patients } & \multicolumn{3}{|c|}{ LQ-Patients } \\
\hline & total & No RT & Neoadjv. RCT & $\overline{\text { Adjv. RT }}$ & total & No RT & Neoadjv. RCT \\
\hline $\mathrm{N}=$ & 182 & $75(41 \%)$ & $89(49 \%)$ & $13(7 \%)$ & 70 & $31(44 \%)$ & $39(56 \%)$ \\
\hline Median Age at first diagnosis [years] & 58 & 59 & 59 & 54 & 57 & 56 & 60 \\
\hline \multicolumn{8}{|l|}{ Grading } \\
\hline G1 & $36(20 \%)$ & $36(48 \%)$ & $4(4 \%)$ & - & $18(25 \%)$ & $18(59 \%)$ & $4(9 \%)$ \\
\hline G2 & $64(35 \%)$ & $23(30 \%)$ & $34(38 \%)$ & $2(18 \%)$ & $26(37 \%)$ & $8(27 \%)$ & $16(42 \%)$ \\
\hline G3 & $78(43 \%)$ & $17(22 \%)$ & $51(57 \%)$ & $9(73 \%)$ & $25(35 \%)$ & $4(14 \%)$ & 19 (49\%) \\
\hline \multicolumn{8}{|l|}{ Medium size of excision } \\
\hline$<10 \mathrm{~cm}$ & $104(57 \%)$ & $48(64 \%)$ & $45(51 \%)$ & $11(82 \%)$ & $43(61 \%)$ & $23(73 \%)$ & $19(50 \%)$ \\
\hline$\geq 10 \mathrm{~cm}$ & $78(43 \%)$ & $28(37 \%)$ & $44(49 \%)$ & $2(18 \%)$ & 27 (39\%) & $8(27 \%)$ & $19(50 \%)$ \\
\hline \multicolumn{8}{|l|}{ Localization } \\
\hline Lower Extremity & $137(75 \%)$ & $54(72 \%)$ & 70 (79\%) & $8(62 \%)$ & $51(73 \%)$ & $20(65 \%)$ & $31(80 \%)$ \\
\hline Upper Extremity & $45(25 \%)$ & $21(28 \%)$ & 19 (21\%) & $5(39 \%)$ & 19 (27\%) & $11(35 \%)$ & $8(20 \%)$ \\
\hline 5-year Survival rate & $149(82 \%)$ & $68(90 \%)$ & $68(76 \%)$ & $10(77 \%)$ & \multirow{2}{*}{\multicolumn{3}{|c|}{ no calculation due to censored data }} \\
\hline 10-year Survival rate & 129 (71\%) & $62(83 \%)$ & $61(68 \%)$ & - & & & \\
\hline Local recurrence & $28(15 \%)$ & $19(10 \%)$ & $7(4 \%)$ & $2(1 \%)$ & $6(9 \%)$ & $5(16 \%)$ & $1(3 \%)$ \\
\hline Metastasis & $42(23 \%)$ & $12(7 \%)$ & $26(14 \%)$ & $4(2 \%)$ & $6(9 \%)$ & $2(7 \%)$ & $3(8 \%)$ \\
\hline
\end{tabular}


Table 3 Major-complications (Clavien-Dindo $\geq 3$ ) of 182 patients with soft tissue sarcoma who underwent sarcoma resection

\begin{tabular}{|c|c|c|c|}
\hline Major-complications & $\begin{array}{l}\begin{array}{l}\text { no RT } \\
(n=75)\end{array} \\
\end{array}$ & neoadjuvant RCT $(n=89)$ & adjuvant RT $(n=13)$ \\
\hline No & 69 (93\%) & $63(72 \%)$ & $12(92 \%)$ \\
\hline Yes & $5(7 \%)$ & $25(28 \%)$ & $1(8 \%)$ \\
\hline Necrosis & & 8 & \\
\hline Wound healing disorders and Infections & 4 & 9 & \\
\hline Thrombosis & 1 & 1 & 1 \\
\hline Bleedings & & 3 & \\
\hline Other & & 4 & \\
\hline \multirow[t]{2}{*}{$p$-value (Fisher-test) } & & $<0.001^{\mathrm{a}}$ & $0.169^{b}$ \\
\hline & & $0.265^{c}$ & \\
\hline
\end{tabular}

$\bar{a}$ neoadjuvant vs. no radiotherapy; ${ }^{\mathrm{b}}$ adjuvant vs. no radiotherapy; ${ }^{\mathrm{c}}$ neoadjuvant vs. adjuvant radiotherapy

global QoL $(\mathrm{H}(3)=7.572, p=0.056)$ and in financial problems $(\mathrm{H}(3)=7.567, p=0.056)$ (Table 5).

\section{Discussion}

In this study we present our results of soft tissue sarcoma patients QoL in a retrospective single-center study and tried to differentiated into different groups in case of radio (chemo) therapy, compared to non-irradiated patients.

Many other retrospective studies, register studies and systematic reviews have examined the role of neo- and adjuvant radio (chemo) therapy in regard to different parameters apart from QoL, such as local control, recurrence rate and

Table 4 Comparing quality of life in two different groups of patients with neoadjuvantor without radiotherapy

\begin{tabular}{|c|c|c|c|}
\hline \multirow[t]{2}{*}{ QLQ-C30 } & \multicolumn{2}{|l|}{ Radiotherapy } & \multirow{2}{*}{$\begin{array}{l}p \text {-value (no } \\
\text { radio- } \\
\text { therapy vs. } \\
\text { neoadjuvant) }\end{array}$} \\
\hline & $\begin{array}{l}\text { no } \\
(n=31) 42 \%\end{array}$ & $\begin{array}{l}\text { neo-adjuvant } \\
(n=39) 52 \%\end{array}$ & \\
\hline Global QoL score & $73.1(65-81)$ & $58.6(51-66)$ & $0.006^{*}$ \\
\hline Physical function score & $88.1(83-94)$ & $68.2(60-76)$ & $<0.001^{*}$ \\
\hline Role function score & $75.2(67-84)$ & $52.9(43-63)$ & $0.002^{*}$ \\
\hline Emotional function score & $80.1(73-87)$ & $65.3(57-74)$ & $0.022^{*}$ \\
\hline Cognitive function score & $88.8(83-95)$ & $80.1(72-88)$ & 0.247 \\
\hline Social function score & $87.6(80-95)$ & $60,9(51-71)$ & $<0.001^{*}$ \\
\hline Fatigue score & $25.9(15-37)$ & $43.4(34-53)$ & $0.023^{*}$ \\
\hline Pain score & $22(13-31)$ & $42.9(31-55)$ & $0.016^{*}$ \\
\hline Insomnia score & $19.3(10-29)$ & $25,4(15-36)$ & 0.505 \\
\hline Appetite loss score & $3.2(-0.4-6.9)$ & $3.9(0.47-7.2)$ & 0.68 \\
\hline Nausea and vomiting score & $1.6(-0-3.5)$ & $1.7(-0.3-3.8)$ & 0.824 \\
\hline Constipation score & $6.4(-1.5-14)$ & $12.2(4-21)$ & 0.151 \\
\hline Diarrhoea score & $6.4(0.6-12)$ & $10.8(4.3-17)$ & 0.286 \\
\hline Dyspnea score & $7.5(2.3-13)$ & $22.8(13-33)$ & $0.034^{*}$ \\
\hline Financial problems score & $11.8(2-22)$ & $31.5(20-44)$ & $0.009^{*}$ \\
\hline
\end{tabular}

* statistically significant $(p<0.05)$. Shown as $\mathrm{Cl}=$ Confidence interval $95 \%$ and lower/upper confidence bounds (Cl (lower-upper)) overall survival, for which the quality of surgical resection seems to be crucially $[11,12]$. Since the first description of Rosenberg [1], the combination of surgery and radiotherapy in soft tissue sarcoma treatment is well established [13]. AlAbsi et al. concluded that delayed surgical resection because of preoperative radiation does not seem to increase the risk of lethal metastatic spread [5]. To date, no differences were found in overall survival, progression free, or local disease control in case of radiotherapy in soft tissue sarcoma treatment. However, recently a meta-analysis suggested that radiotherapy is associated with lower long-term mortality [14]. In our patient population, $49 \%$ of all included patients were additionally treated with neoadjuvant RCT. Other studies reported much lower radiation rates ranging from $9 \%$ in contrast to much higher rates up to $100 \%$ for planned sarcoma excision $[15,16]$.

In our study, we first investigated postoperative major complications of pre- and postoperative radio (chemo) therapy in the treatment of soft tissue sarcoma. In each case, we analyzed the kind of major complication and classified it to postoperative major complication rate or not. In the case of postoperative RT and postoperative major complications, the time period between surgery and RT were very close or classification clear. Major and overall complication rates were not significantly different in one study (major: 28.2 vs. $25.2 \%, p=0.69$; overall 35.2 vs. $33.2 \%, p=0.83$ ) [15]. However, we observed statistically significant differences in major complications rates after neoadjuvant RCT (28 vs. $7 \%, p<0.001$ ), but not after CT only ( 0 vs. $7 \%, p=0.13)$. The reason for differences in complication rates of irradiated patients remains unclear. Nussbaum et al. reported on a very large study population (785 Patients) with a small radiation rate of $9 \%$ (our data $49 \%$ ) and focused on short-term (30 days) morbidity and mortality as well as on retroperitoneal sarcoma [15]. Meric et al. found a 50\% increase in wound complications among patients treated with preoperative RT [17]. In case of postoperative RT, we 


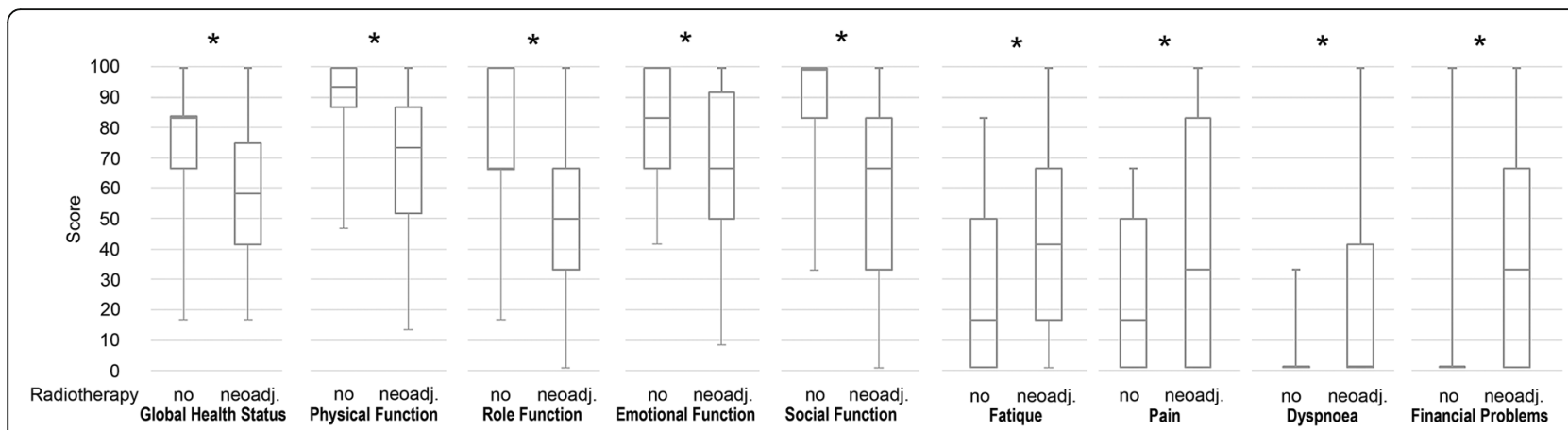

Fig. 2 Quality of life scores of extremity soft tissue sarcoma patients who underwent preoperative RCT and surgery or no RT (and only surgery). x-pivot: $95 \%$ Confidence Interval of Quality of Life score. Global: global quality of life score; physical: physical functioning score; role: role functioning score; emotional: emotional functioning score; social: social functioning score; fatigue: fatigue score; dyspnoea: dyspnoea score; pain: pain score. Functioning scores: best score: 100. Pain Score: best score: 0 . Figure showing only the significantly different scores/items

noticed major complication rates of $8 \%$ (vs. $7 \%, p=$ 0.265). In accordance with our results, Miller et al. reported higher wound complication rates after neoadjuvant than after postoperative RT (35 vs. $17 \%, p=0.01$ ) [18]. In a prospective trial by O'Sullivan et al., 190 patients with extremity soft tissue sarcoma were randomly allocated to either preoperative or postoperative RT. Acute wound complications were significantly higher in the neoadjuvant group (35 vs. $17 \%$ in the postoperative group) [19].

Major complication rates which need additional operations or in-hospital treatment are serious adverse events. Even though the levels of these complication rates are in accordance with the literature, all efforts should be made to further decrease those levels. However, the risk of such major complications may not only be related RT, but possibly even more to the patient [20] and individual tumor characteristics [21] and tumor specific molecular mechanisms [22, 23]. In addition, different surgical resection modes (wide excision or compartment resection in contrast to simple resection [24]), differences in preoperative treatments, and various localizations (maybe affecting of neurovascular structures bones or joints) of soft tissue sarcomas may play a role in major complication rates. Furthermore, specific technical RT parameters such as total dose, fraction size, treatment volume and RT techniques, which could not be analyzed within this study due to incomplete data, may play a pivotal role for the risk of developing major complications.

Our study also had some additional limitations according to other investigations in the literature [25]. First, we included 182 patients with different tumor localizations, different clinical and/or pathological statuses and various soft tissue sarcoma subtypes, because individual subtype analyses would have resulted in very small group sizes, rendering statistical analysis impossible [26]. In general, the larger and more malignant a STS is, the more likely the patient is to receive chemotherapy and/or radiotherapy. Conversely, small superficial tumors are unlikely to receive CT or RT. In contrast to a prospective analysis, retrospective data analysis allows no stratification for example regarding tumor size. Possibly prospective studies with stratification regarding tumor size (and/or grading) might show that the very tumors which did not need chemotherapy or RT are at the same time more eligible to complete surgical resection and thus might result in quicker recovery with less complications and as a consequence yielding better QoL. Furthermore there are wellrecognized limitations of retrospective studies per se, e. g. dependence on the limited medical data records in particular concerning patients who are deceased or lost to follow-up, no measurement of late toxicity effects and selection bias [27]. Since patients were contacted after completion of their therapy (median interval: 4.98 years), we were only able to report final outcome QoL not during the mean time / whilst being on therapy, which might also influence the final and ex post QoL estimation by the patients. Furthermore, because of the retrospective analyses we have a lack of baseline evaluation of QoL before treatment with or without radio (chemo)therapy. It is possible, that patient candidate to radiation therapy could have had a worse QoL baseline due to more advanced disease. Finally, the sample size of this series still is not very large, with is attributed to the rarity of this disease. Nevertheless, given the lack of literature on QoL outcomes after radio (chemo) therapy and the rarity of these tumors, we hope this study might add new information regarding the possible impact of neo -/adjuvant radio (chemo) therapy on QoL in soft tissue sarcoma patients, even it is not possible to discriminate whether radiation therapy could really had an impact or not in retrospective studies.

To our best knowledge there are only a few investigations of QoL in soft tissue sarcomas [28]: the purpose of the study by Parsons et al., e. g., was to investigate rehabilitation aims of patients with soft tissue sarcoma and chronic disability using the World Health Organization's 
Table 5 Stratification of Disease-Control (Disease-free vs. Recurrence/Metastasis). Analysis with Kruskal-Wallis H-Test. Values given as Mean Rank

\begin{tabular}{|c|c|c|c|c|c|}
\hline QLQ-C30 & $\begin{array}{l}\text { No RT, Disease- } \\
\text { free }\end{array}$ & $\begin{array}{l}\text { No RT, Recurrence / } \\
\text { Metastasis }\end{array}$ & $\begin{array}{l}\text { Neoadj. RT, Disease- } \\
\text { free }\end{array}$ & $\begin{array}{l}\text { Neoadj. RT, Recurrence / } \\
\text { Metastasis }\end{array}$ & $\begin{array}{l}\mathrm{H} \text { - and, } p- \\
\text { value }\end{array}$ \\
\hline Global QoL score & 42.96 & 42.33 & 30.10 & 25.88 & $\begin{array}{l}H(3)=7.572 \\
p=0.056\end{array}$ \\
\hline Physical function score & 44.92 & 44.33 & 26.94 & 27.50 & $\begin{array}{l}H(3)= \\
13.787 \\
p=0.003^{*}\end{array}$ \\
\hline \multicolumn{3}{|l|}{ Pairwise comparison } & \multicolumn{3}{|l|}{$\mathrm{H}$ - and $p$-value } \\
\hline \multicolumn{3}{|c|}{ Neoadj. RT + Disease-free vs. No RT + Disease free } & \multicolumn{3}{|l|}{$\mathrm{H}=17.979, p=0.003^{*}$} \\
\hline \multicolumn{3}{|c|}{ Neoadj. RT + Disease-free vs. Neoadj. RT + Recurrence/Metastasis } & \multicolumn{3}{|l|}{$H=0.559, p=1.00$} \\
\hline \multicolumn{3}{|c|}{ Neoadj. RT + Disease-free vs. No RT + Recurrence/Metastasis } & \multicolumn{3}{|l|}{$H=17.392, p=0.28$} \\
\hline \multicolumn{3}{|c|}{ Neoadj. RT + Recurrence/Metastasis vs. No RT + Recurrence/Metastasis } & \multicolumn{3}{|l|}{$H=16.833, p=1.00$} \\
\hline \multicolumn{3}{|c|}{ Neoadj. RT + Recurrence/Metastasis vs. No RT + Disease-free } & \multicolumn{3}{|l|}{$H=17.420, p=0.616$} \\
\hline \multicolumn{3}{|c|}{ No RT + Recurrence/Metastasis vs. No RT + Disease-free } & \multicolumn{3}{|l|}{$\mathrm{H} 0.587, p=1.00$} \\
\hline Role function score & 43.44 & 45.17 & 30.00 & 19.50 & $\begin{array}{l}H(3)= \\
10.696 \\
p=0.01^{*}\end{array}$ \\
\hline \multicolumn{3}{|l|}{ Pairwise comparison } & \multicolumn{3}{|l|}{$\mathrm{H}$ - and $p$-value } \\
\hline \multicolumn{3}{|c|}{ Neoadj. RT + Disease-free vs. RT + Disease free } & \multicolumn{3}{|l|}{$H=13.440, p=0,05^{*}$} \\
\hline \multicolumn{3}{|c|}{ Neoadj. RT + Disease-free vs. Neoadj. RT + Recurrence/Metastasis } & \multicolumn{3}{|l|}{$H=10.500, p=1.00$} \\
\hline \multicolumn{3}{|c|}{ Neoadj. RT + Disease-free vs. No RT + Recurrence/Metastasis } & \multicolumn{3}{|l|}{$H=15.167, p=0.50$} \\
\hline \multicolumn{3}{|c|}{ Neoadj. RT + Recurrence/Metastasis vs. No RT + Recurrence/Metastasis } & \multicolumn{3}{|l|}{$\mathrm{H} 25.667, p=0.27$} \\
\hline \multicolumn{3}{|c|}{ Neoadj. RT + Recurrence/Metastasis vs. No RT + Disease-free } & \multicolumn{3}{|l|}{$\mathrm{H}=23.940, p=0.15$} \\
\hline \multicolumn{3}{|c|}{ No RT + Recurrence/Metastasis vs. No RT + Disease-free } & \multicolumn{3}{|l|}{$H=-1.727, p=1.00$} \\
\hline Emotional function score & 40.88 & 44.92 & 31.73 & 20.75 & $\begin{array}{l}H(3)=6.468 \\
p=0.09\end{array}$ \\
\hline Cognitive function score & 37.46 & 39.83 & 33.24 & 28.38 & $\begin{array}{l}H(3)=1.668 \\
p=0,64\end{array}$ \\
\hline Social function score & 45.58 & 42.83 & 27.68 & 19.38 & $\begin{array}{l}H(3)= \\
16.108 \\
p=0.001^{*}\end{array}$ \\
\hline \multicolumn{3}{|l|}{ Pairwise comparison } & $\mathrm{H}$ - and $p$-value & & \\
\hline Neoadj. RT + Disease-free & vs. RT + Disease fre & & $H=17.904, p=0.002^{*}$ & & \\
\hline Neoadj. RT + Disease-free & vs. Neoadj. RT + Re & urrence/Metastasis & $H=8.301, p=1.00$ & & \\
\hline Neoadj. RT + Disease-free & vs. No RT + Recurre & ce/Metastasis & $H=15.157, p=0.45$ & & \\
\hline Neoadj. RT + Recurrence/N & Metastasis vs. No R & + Recurrence/Metastasis & $H=23.458, p=0.35$ & & \\
\hline Neoadj. RT + Recurrence/N & hetastasis vs. No R & + Disease-free & $H=26.205, p=0.06$ & & \\
\hline No RT + Recurrence/Metas & tasis vs. No RT + D & ease-free & $\mathrm{H} 2.747, p=1.00$ & & \\
\hline Fatigue score & 27.50 & 24.42 & 36.57 & 44.38 & $\begin{array}{l}H(3)=5.969 \\
p=0.11\end{array}$ \\
\hline Pain score & 28.52 & 29.92 & 39.09 & 48.38 & $\begin{array}{l}H(3)=6.598 \\
p=0.08\end{array}$ \\
\hline Insomnia score & 33.42 & 33.25 & 33.79 & 57.75 & $\begin{array}{l}H(3)=6.665 \\
p=0.08\end{array}$ \\
\hline Appetite loss score & 35.20 & 31.00 & 36.03 & 31.00 & $\begin{array}{l}H(3)=1.589 \\
p=0.66\end{array}$ \\
\hline $\begin{array}{l}\text { Nausea and vomiting } \\
\text { score }\end{array}$ & 34.72 & 37.67 & 34.09 & 40.50 & $\begin{array}{l}H(3)=2.020 \\
p=0.56\end{array}$ \\
\hline Constipation score & 33.30 & 29.00 & 35.93 & 46.75 & $\begin{array}{l}H(3)=4.962 \\
p=0.17\end{array}$ \\
\hline
\end{tabular}


Table 5 Stratification of Disease-Control (Disease-free vs. Recurrence/Metastasis). Analysis with Kruskal-Wallis H-Test. Values given as Mean Rank (Continued)

\begin{tabular}{|c|c|c|c|c|c|}
\hline Diarrhoea score & 32.20 & 33.67 & 35.11 & 45.12 & $\begin{array}{l}H(3)=2.946 \\
P=0.40\end{array}$ \\
\hline Dyspnea score & 29.50 & 33.50 & 36.16 & 61.75 & $\begin{array}{l}H(3)= \\
13.168 \\
p=0.004^{*}\end{array}$ \\
\hline \multicolumn{3}{|l|}{ Pairwise comparison } & \multicolumn{3}{|c|}{$\mathrm{H}$ - and $p$-value } \\
\hline \multicolumn{3}{|c|}{ Neoadj. RT + Disease-free vs. RT + Disease free } & \multicolumn{3}{|c|}{$H=-6.662, p=0.78$} \\
\hline \multicolumn{3}{|c|}{ Neoadj. RT + Disease-free vs. Neoadj. RT + Recurrence/Metastasis } & \multicolumn{3}{|c|}{$H=-25.588, p=0.02^{*}$} \\
\hline \multicolumn{3}{|c|}{ Neoadj. RT + Disease-free vs. No RT + Recurrence/Metastasis } & \multicolumn{3}{|c|}{$H=-2.662, p=1.00$} \\
\hline \multicolumn{3}{|c|}{ Neoadj. RT + Recurrence/Metastasis vs. No RT + Recurrence/Metastasis } & \multicolumn{3}{|c|}{$H=-28.250, p=0.05^{*}$} \\
\hline \multicolumn{3}{|c|}{ Neoadj. RT + Recurrence/Metastasis vs. No RT + Disease-free } & \multicolumn{3}{|c|}{$H=-32.250, p=0.002^{*}$} \\
\hline \multicolumn{3}{|c|}{ No RT + Recurrence/Metastasis vs. No RT + Disease-free } & \multicolumn{3}{|c|}{$H=-4.000, p=1.00$} \\
\hline Financial problems score & 28.42 & 30.83 & 39.21 & 46.62 & $\begin{array}{l}H(3)=7.567 \\
p=0.056\end{array}$ \\
\hline
\end{tabular}

* statistically significant $(p<0.05)$. Pairwise Comparison using Mann Whitney U-Test after Bonferroni procedure

(WHO) international classification of functioning, disability, and health (ICF). There was the strongest support for complex decongestive physiotherapy and aerobic exercise interventions [29]. In 2006 Schreiber et al. evaluated function- and health-related quality of life in 100 extremity soft tissue sarcoma patients, using life orientation test (LOT), musculoskeletal tumor society rating scale (MSTS), reintegration to normal living index (RNL), and Toronto extremity salvage score (TESS). Restriction in participation of life roles and situations has the greatest effect on these patients [30]. Patients' QoL (investigated with RAND-36) after hyperthermic isolated limb perfusion for locally advanced extremity soft tissue sarcoma was significantly worse in physical functioning comparing to the healthy Dutch population [8]. Investigations of QoL after compartimental resection for subfascial extremity soft tissue sarcoma (using EORTC Score C30) showed decreased QoL scores in all dimensions, compared to a normal population [31]. Reichardt et al. and Coens et al. investigated the HRQoL of patients with soft tissue sarcoma and chemotherapy [32,33]. Sachsenmaier et al. created a new questionnaire. Based on this questionnaire, they were able to identify risk factors for poor emotional outcome after therapy, related to patients' physical, psychological and social situation [34]. Xu et al. reported in 2017 a better functional outcome and QoL using Chinese MSTS scoring system for patients receiving limb-salvage surgeries than those undergoing amputation surgeries [35].

Overall, literature about QoL of patients with soft tissue sarcoma and radiotherapy is very rare. To the best of our knowledge there have been no HRQoL investigations of patients with soft tissue sarcoma and radiotherapy (except hyperthermic limb perfusion) reported in the literature until today. Some studies address the functional outcome after surgery of extremity soft tissue sarcomas in scores, but the scales vary between the reports. A review of 145 patients which were treated with limited surgery and postoperative radiotherapy showed that $20 \%$ of patients developed contracture, $19 \%$ significant edema, $7 \%$ required the use of a crutch, and 6\% experienced a bone fracture [36].

The EORTC QLQ-C30 used in this study is well established and validated in cancer [10,37]. It is multidimensional, incorporating all aspects of daily life, as well as being subjective [38]. Comparing the group of preoperative RCT vs. non-irradiated patients shows that global QoL, physical, role, and social functioning, emotional functioning, fatigue, pain and financial problem scores were significantly worse in the neoadjuvant radiotherapy group, compared to the group without RT (Table 4 and Fig. 2). It is known that RT in cancer therapy affected cancer patients' QoL negatively [39]. In contrast to Yucel et al. [39], we did not measure a restoration of pre-treatment HRQoL after completion off the RT. This is in accordance with the study of Bansal at al., evaluating 45 patients with head and neck cancer [40, 41]. After completion of sarcoma treatment, we still observed significantly worse scores for global QoL, physical, role, and social functioning, emotional functioning, fatigue, pain and financial problems.

RT itself is administered over weeks. This is also a probable reason for financial difficulties, because patients were possibly far away from home and off work for a long time [38]. It is also possible that the combination of RT and intensive CT causes the reduced HRQoL scores. However, some studies have shown reduced QoL when radiotherapy and chemotherapy were combined compared to radiotherapy alone [42]. Further analysis of CT effects alone (without RT) would be helpful.

Disease status of the patients who answered the questionnaires could have been an influence of QoL. Stratification into four groups (No RT Disease-free, Neoadj. RT 
Disease-free, No RT Recurrence/Metastasis, Neoadj. RT Recurrence/Metastasis) found significant reduced scores of physical functioning, role functioning and social functioning in the group of disease-free patients with neoadjuvant RT compared to the disease-free patients without RT and a strong trend for global QoL and financial problems without statistical significance. A direct correlation of QoL to the initial treatment is despite these results difficult, because the number of patients living with recurrence, metastasis or disease-control was differently and a further differentiation between patients with recurrence and patients with metastasis was in our collective not possible.

Nevertheless, further analysis and especially prospective studies of QoL with stratifications of disease control in STS patients with and without radiotherapy are necessary to evaluate a directly correlation of treatment modalities in primary disease and QoL.

\section{Conclusion}

We present our results of soft tissue sarcoma patients' HRQoL in the case of RT in a retrospective singlecenter study. We observed statistically significant differences in major complications rates after neoadjuvant RCT ( 28 vs. $7 \%, p<0.001)$. In the case of postoperative RT, we observed similar major complication rates as compared to no-RT ( $8 \%$ vs. $7 \%$, respectively; $p=0.265$ ). We observed that global QoL, physical, role, and social functioning, emotional functioning, fatigue, pain and financial problem scores were significantly worse in the neoadjuvant RCT group, compared to the group without RT. Given the lack of literature on HRQoL outcomes after RT in soft tissue sarcoma and the rarity of these tumors, we think this study may help to reduce this gap of knowledge, even there are limitations especially because of the retrospective analysis and a direct conclusion of the primary treatment and the QoL are not possible.

\section{Abbreviations}

CT: Chemotherapy; EORTC: European Organization for Research and Treatment of Cancer; HRQoL: Health related quality of life; MSTS: Musculoskeletal tumor society rating scale; QoL: Quality of life; RCT: Radiochemotherapy; RNL: Reintegration to normal index; RT: Radiotherapy; STS: Soft tissue sarcoma; TESS: Toronto extremity salvage score; UPS: Undifferentiated pleomorphic sarcoma; WHO: World Health Organization

\section{Acknowledgements}

The present work was performed in fulfillment of the requirements for obtaining the degree "Dr. med." for Sebastian Sterzinger.

\section{Authors'contributions}

Study concepts: JPB, RG. Study design: JPB, RG. Data acquisition: RG, SS, NV AA, SS. Quality control of data and algorithms: JPB, RG, WH, AA. Data analysis and interpretation: JPB, RG, SS, SS, WH, RG, AA, REH. Statistical analysis: RG, SS. Manuscript preparation: RG, JB. Manuscript editing: RG, SS. Manuscript review: JPB, SS, NV, WH, RG, AA, AA, REH. All authors read and approved the final manuscript.

\section{Availability of data and materials}

The datasets used and analyzed during the current study are available from the corresponding author on reasonable request.

\section{Ethics approval and consent to participate}

Research has been performed in accordance with the Declaration of Helsinki. Each Patient has been signed a consent of participate.

\section{Consent for publication}

Not applicable.

\section{Competing interests}

The authors declare that they have no competing interests.

\section{Author details}

${ }^{1}$ Department of Plastic and Hand Surgery, Comprehensive Cancer Center, Universitiy Hospital of Erlangen, Friedrich-Alexander-University

Erlangen-Nürnberg (FAU), Erlangen, Germany. ${ }^{2}$ Present Address: Department of Plastic Surgery, Hand and Burn Surgery, University Hospital of Aachen, RWTH University of Aachen, Aachen, Germany. ${ }^{3}$ Department of Radiation Oncology, Comprehensive Cancer Center, University Hospital of Erlangen, Friedrich-Alexander-University Erlangen-Nürnberg (FAU), Erlangen, Germany. ${ }^{4}$ Department of Surgery, Comprehensive Cancer Center, University Hospital of Erlangen, Friedrich-Alexander-University Erlangen-Nürnberg (FAU), Erlangen, Germany. ${ }^{5}$ Department of Pathology, Comprehensive Cancer Center, University Hospital of Erlangen, Friedrich-Alexander-University Erlangen-Nürnberg (FAU), Erlangen, Germany.

Received: 31 May 2019 Accepted: 22 October 2019

Published online: 08 November 2019

\section{References}

1. Rosenberg SA, Tepper J, Glatstein E, Costa J, Baker A, Brennan M, et al. The treatment of soft-tissue sarcomas of the extremities: prospective randomized evaluations of (1) limb-sparing surgery plus radiation therapy compared with amputation and (2) the role of adjuvant chemotherapy. Ann Surg. 1982;196(3):305-15.

2. Group ESESNW. Soft tissue and visceral sarcomas: ESMO Clinical Practice Guidelines for diagnosis, treatment and follow-up. Ann Oncol. 2014; 25(Suppl 3):iii102-12.

3. Kachare SD, Brinkley J, Vohra NA, Zervos EE, Wong JH, Fitzgerald TL. Radiotherapy associated with improved survival for high-grade sarcoma of the extremity. J Surg Oncol. 2015;112(4):338-43.

4. Albertsmeier M, Rauch A, Roeder F, Hasenhutl S, Pratschke S, Kirschneck M, et al. External beam radiation therapy for resectable soft tissue sarcoma: a systematic review and meta-analysis. Ann Surg Oncol. 2018;25(3):754-67.

5. Al-Absi E, Farrokhyar F, Sharma R, Whelan K, Corbett T, Patel M, et al. A systematic review and meta-analysis of oncologic outcomes of pre- versus postoperative radiation in localized resectable soft-tissue sarcoma. Ann Surg Oncol. 2010;17(5):1367-74.

6. Dickie $\mathrm{Cl}$, Haas R, O'Sullivan B. Adjuvant radiation for soft tissue sarcomas. Am Soc Clin Oncol Educ Book / ASCO Am Soc Clin Oncol Meeting. Am Soc Clin Oncol Educ Book. 2015;35:e634-42. https://doi.org/10.14694/EdBook_AM.2015. $35 . e 634$

7. Hurmuzlu M, Aarstad HJ, Aarstad AK, Hjermstad MJ, Viste A. Health-related quality of life in long-term survivors after high-dose chemoradiotherapy followed by surgery in esophageal cancer. Dis Esophagus. 2011:24(1):39-47.

8. Thijssens KM, Hoekstra-Weebers JE, van Ginkel RJ, Hoekstra HJ. Quality of life after hyperthermic isolated limb perfusion for locally advanced extremity soft tissue sarcoma. Ann Surg Oncol. 2006;13(6):864-71.

9. Coindre JM. Grading of soft tissue sarcomas: review and update. Arch Pathol Lab Med. 2006:130(10):1448-53.

10. Aaronson NK, Ahmedzai S, Bergman B, Bullinger M, Cull A, Duez NJ, et al. The European Organization for Research and Treatment of Cancer QLQ-C30: a quality-of-life instrument for use in international clinical trials in oncology. Jatl Cancer Inst. 1993:85(5):365-76.

11. Rastrelli M, Tropea S, Basso U, Roma A, Maruzzo M, Rossi CR. Soft tissue limb and trunk sarcomas: diagnosis, treatment and follow-up. Anticancer Res. 2014;34(10):5251-62. 
12. Kapoor T, Banuelos J, Adabi K, Moran SL, Manrique OJ. Analysis of clinical outcomes of upper and lower extremity reconstructions in patients with soft-tissue sarcoma. J Surg Oncol. 2018;118(4):614-20.

13. Fairweather M, Keung E, Raut CP. Neoadjuvant Therapy for Soft-Tissue Sarcomas. Oncology (Williston Park, NY). 2016;30(1):99-106.

14. Qu X, Lubitz CC, Rickard J, Bergeron SG, Wasif N. A meta-analysis of the association between radiation therapy and survival for surgically resected soft-tissue sarcoma. Am J Clin Oncol. 2018;41(4):348-56.

15. Nussbaum DP, Speicher PJ, Gulack BC, Ganapathi AM, Keenan JE, Stinnett SS, et al. The effect of neoadjuvant radiation therapy on perioperative outcomes among patients undergoing resection of retroperitoneal sarcomas. Surg Oncol. 2014;23(3):155-60.

16. Haas RL, Miah AB, LePechoux C, DeLaney TF, Baldini EH, Alektiar K, et al. Preoperative radiotherapy for extremity soft tissue sarcoma; past, present and future perspectives on dose fractionation regimens and combined modality strategies. Radiother Oncol. 2016;119(1):1421.

17. Meric F, Milas M, Hunt KK, Hess KR, Pisters PW, Hildebrandt G, et al. Impact of neoadjuvant chemotherapy on postoperative morbidity in soft tissue sarcomas. J Clin Oncol. 2000;18(19):3378-83.

18. Miller ED, Xu-Welliver M, Haglund KE. The role of modern radiation therapy in the management of extremity sarcomas. J Surg Oncol. 2015;111(5):599-603.

19. O'Sullivan B, Davis AM, Turcotte R, Bell R, Catton C, Chabot P, et al. Preoperative versus postoperative radiotherapy in soft-tissue sarcoma of the limbs: a randomised trial. Lancet (London, England). 2002;359(9325):2235-41.

20. Montgomery C, Harris J, Siegel E, Suva L, Wilson M, Morell S, et al. Obesity is associated with larger soft-tissue sarcomas, more surgical complications, and more complex wound closures (obesity leads to larger soft-tissue sarcomas). J Surg Oncol. 2018;118(1):184-91.

21. Kaminska K, Szczylik C, Bielecka ZF, Bartnik E, Porta C, Lian F, et al. The role of the cell-cell interactions in cancer progression. J Cell Mol Med. 2015;19(2):283-96.

22. Alias C, Rocchi L, Ribatti D, Caraffi S, D'Angelo A, Perris R, et al. MMPs and angiogenesis affect the metastatic potential of a human vulvar leiomyosarcoma cell line. J Cell Mol Med. 2015;19(9):2098-107.

23. Bianchi G, Sambri A, Righi A, Dei Tos AP, Picci P, Donati D. Histology and grading are important prognostic factors in synovial sarcoma. Eur J Surg Oncol. 2017:43(9):1733-9.

24. Iwata S, Araki A, Funatsu H, Yonemoto T, Kamoda H, Itami M, et al. Optimal surgical margin for infiltrative soft tissue sarcomas: assessing the efficacy of excising beyond the infiltration. J Surg Oncol. 2018;118(3):525-31.

25. Cassidy RJ, Indelicato DJ, Gibbs CP, Scarborough MT, Morris CG, Zlotecki RA. Function preservation after conservative resection and radiotherapy for softtissue sarcoma of the distal extremity: utility and application of the Toronto Extremity Salvage Score (TESS). Am J Clin Oncol. 2016;39(6):600-3.

26. Abatzoglou S, Turcotte RE, Adoubali A, Isler MH, Roberge D. Local recurrence after initial multidisciplinary management of soft tissue sarcoma: is there a way out? Clin Orthop Relat Res. 2010;468(11):3012-8.

27. Harriman SL, Patel J. When are clinical trials registered? An analysis of prospective versus retrospective registration. Trials. 2016;17(1):187.

28. Sugarbaker PH, Barofsky I, Rosenberg SA, Gianola FJ. Quality of life assessment of patients in extremity sarcoma clinical trials. Surgery. 1982; 91(1):17-23

29. Parsons JA, Davis AM. Rehabilitation and quality-of-life issues in patients with extremity soft tissue sarcoma. Curr Treat Options in Oncol. 2004;5(6):477-88.

30. Schreiber D, Bell RS, Wunder JS, O'Sullivan B, Turcotte R, Masri BA, et al. Evaluating function and health related quality of life in patients treated for extremity soft tissue sarcoma. Qual Life Res. 2006;15(9):1439-46.

31. Peiper M, Matthaei H, Bolke E, Zurakowski D, Orth K, Heinecke A, et al. Compartmental resection for subfascial extremity soft tissue sarcoma and quality of life in long-term sunvivors. Wien Klin Wochenschr. 2011;123(15-16):488-95.

32. Reichardt P, Leahy M, Garcia Del Muro X, Ferrari S, Martin J, Gelderblom H, et al. Quality of life and utility in patients with metastatic soft tissue and bone sarcoma: the sarcoma treatment and burden of illness in North America and Europe (SABINE) study. Sarcoma. 2012;2012:740279.

33. Coens C, van der Graaf WT, Blay JY, Chawla SP, Judson I, Sanfilippo R, et al. Health-related quality-of-life results from PALETTE: a randomized, doubleblind, phase 3 trial of pazopanib versus placebo in patients with soft tissue sarcoma whose disease has progressed during or after prior chemotherapya European organization for research and treatment of cancer soft tissue and bone sarcoma group global network study (EORTC 62072). Cancer. 2015;121(17):2933-41.
34. Sachsenmaier SM, Ipach I, Kluba T. Quality of life, physical and mental status and contentment of patients with localized soft tissue or bone sarcoma: a questionnaire analysis. Orthop Rev. 2015;7(2):5920.

35. Xu L, Li X, Wang Z, Xiong J, Wang S. Functional evaluation for patients with lower extremity sarcoma: application of the Chinese version of musculoskeletal tumor society scoring system. Health Qual Life Outcomes. 2017;15(1):107.

36. Stinson SF, DeLaney TF, Greenberg J, Yang JC, Lampert MH, Hicks JE, et al. Acute and long-term effects on limb function of combined modality limb sparing therapy for extremity soft tissue sarcoma. Int J Radiat Oncol Biol Phys. 1991;21(6):1493-9.

37. van Leeuwen M, Husson O, Alberti P, Arraras Jl, Chinot OL, Costantini A, et al. Understanding the quality of life (QOL) issues in survivors of cancer: towards the development of an EORTC QOL cancer survivorship questionnaire. Health Qual Life Outcomes. 2018;16(1):114.

38. Srivastava A, Vischioni B, Fiore MR, Vitolo V, Fossati P, lannalfi A, et al. Quality of life in patients with chordomas/chondrosarcomas during treatment with proton beam therapy. J Radiat Res. 2013;54(Suppl 1):i43-8.

39. Yucel B, Akkas EA, Okur Y, Eren AA, Eren MF, Karapinar H, et al. The impact of radiotherapy on quality of life for cancer patients: a longitudinal study. Support Care Cancer. 2014;22(9):2479-87.

40. Bansal M, Mohanti BK, Shah N, Chaudhry R, Bahadur S, Shukla NK. Radiation related morbidities and their impact on quality of life in head and neck cancer patients receiving radical radiotherapy. Qual Life Res. 2004;13(2):481-8.

41. Semrau S, Haderlein M, Schmidt D, Lell M, Wolf W, Waldfahrer F, et al. Single-cycle induction chemotherapy followed by chemoradiotherapy or surgery in patients with head and neck cancer: what are the best predictors of remission and prognosis? Cancer. 2015;121(8):1214-22

42. Woo B, Dibble SL, Piper BF, Keating SB, Weiss MC. Differences in fatigue by treatment methods in women with breast cancer. Oncol Nurs Forum. 1998. 25(5):915-20.

\section{Publisher's Note}

Springer Nature remains neutral with regard to jurisdictional claims in published maps and institutional affiliations.

\section{Ready to submit your research? Choose BMC and benefit from:}

- fast, convenient online submission

- thorough peer review by experienced researchers in your field

- rapid publication on acceptance

- support for research data, including large and complex data types

- gold Open Access which fosters wider collaboration and increased citations

- maximum visibility for your research: over $100 \mathrm{M}$ website views per year

At BMC, research is always in progress.

Learn more biomedcentral.com/submissions 